\title{
A 2015 International Geomagnetic Reference Field (IGRF) candidate model based on Swarm's experimental absolute magnetometer vector mode data
}

\author{
Pierre Vigneron ${ }^{1}$, Gauthier Hulot ${ }^{1 *}$, Nils Olsen², Jean-Michel Léger ${ }^{3}$, Thomas Jager $^{3}$, Laura Brocco ${ }^{1}$, Olivier Sirol ${ }^{1}$,
} Pierdavide Coïsson ${ }^{1}$, Xavier Lalanne ${ }^{1}$, Arnaud Chulliat ${ }^{4}$, François Bertrand ${ }^{3}$, Axel Boness ${ }^{3}$ and Isabelle Fratter ${ }^{5}$

\begin{abstract}
Each of the three satellites of the European Space Agency Swarm mission carries an absolute scalar magnetometer (ASM) that provides the nominal $1-\mathrm{Hz}$ scalar data of the mission for both science and calibration purposes. These ASM instruments, however, also deliver autonomous $1-\mathrm{Hz}$ experimental vector data. Here, we report on how ASM-only scalar and vector data from the Alpha and Bravo satellites between November 29, 2013 (a week after launch) and September 25, 2014 (for on-time delivery of the model on October 1, 2014) could be used to build a very valuable candidate model for the 2015.0 International Geomagnetic Reference Field (IGRF). A parent model was first computed, describing the geomagnetic field of internal origin up to degree and order 40 in a spherical harmonic representation and including a constant secular variation up to degree and order 8 . This model was next simply forwarded to epoch 2015.0 and truncated at degree and order 13. The resulting ASM-only 2015.0 IGRF candidate model is compared to analogous models derived from the mission's nominal data and to the now-published final 2015.0 IGRF model. Differences among models mainly highlight uncertainties enhanced by the limited geographical distribution of the selected data set (essentially due to a lack of availability of data at high northern latitude satisfying nighttime conditions at the end of the time period considered). These appear to be comparable to differences classically observed among IGRF candidate models. These positive results led the ASM-only 2015.0 IGRF candidate model to contribute to the construction of the final 2015.0 IGRF model.
\end{abstract}

Keywords: Swarm; IGRF; Absolute vector magnetometer

\section{Background}

Every 5 years, the International Association of Geomagnetism and Aeronomy (IAGA) releases a new update of what is known as the International Geomagnetic Reference Field (IGRF) model. This model is in fact a series of models providing snapshots of the past main geomagnetic field every 5 years since 1900. A secular variation model is also provided, describing the constant trend predicted for the evolution of the field over the 5 years following the update (see, e.g., Macmillan and Finlay (2011), for a general introduction to IGRF models, and Hulot et al. (2015a), for more

\footnotetext{
* Correspondence: gh@ipgp.fr

${ }^{1}$ Institut de Physique du Globe de Paris, Sorbonne Paris Cité, Université Paris Diderot, CNRS, F-75005 Paris, France

Full list of author information is available at the end of the article
}

background on geomagnetic field modeling). Every time a new release is due, a dedicated task force is set up by IAGA and an open call issued, inviting the scientific community to produce and propose so-called candidate models. The latest call, issued in May 2014, requested candidate models for the 2015.0 IGRF model and for the 2015.0-2020.0 predictive IGRF secular variation model. As is standard practice, it also called for candidate models for the a posteriori 2010.0 Definitive Geomagnetic Reference Field (DGRF) model, meant to replace the 2010.0 IGRF model provided with the previous 2010 IGRF release (Finlay et al. 2010). All candidate models were requested by the deadline of October 1, 2014. The present paper reports on the production of a 2015.0 IGRF candidate model in response to this call. This model was built 
from a unique set of data acquired by absolute scalar magnetometers (ASM) running an experimental vector mode on board the satellites of the European Space Agency (ESA) Swarm mission. For reasons later explained, no candidate predictive IGRF secular variation model for the interval 2015.0-2020.0 was proposed, but only a test model. As the Swarm mission was only launched on November 22, 2013, no DGRF 2010.0 candidate model was proposed, either.

The Swarm mission is the fifth Earth Explorer Mission in the Living Planet Programme of ESA (see Friis-Christensen et al. (2006), for general background information). It consists of a constellation of three nominally identical satellites. Two (Alpha and Charlie) fly almost side by side on lowaltitude polar orbits (inclination of $87.4^{\circ}$, longitude separation of $1.4^{\circ}$, altitude of slightly more than $470 \mathrm{~km}$ above a mean radius of $a=6371.2 \mathrm{~km}$ in September 2014). The third satellite (Bravo) is on a slightly different orbit since April 2014 (88 inclination and slightly more than $520 \mathrm{~km}$ altitude in September 2014) to allow for a progressive local time separation with respect to Alpha and Charlie. The magnetometer payload consists of three instruments, located on a boom to minimize mutual interferences and perturbations caused by the satellite itself (see Fig. 1). Two are mounted on a common rigid optical bench: a vector fluxgate magnetometer (VFM), which measures the direction and relative strength of the magnetic field, and a three-head star tracker (STR), which provides the attitude information needed to transform the vector readings to a known terrestrial coordinate frame. The third instrument is the ASM. It is located at the tip of the satellite's boom, $2 \mathrm{~m}$ further away, and its nominal role is to provide absolute measurements of the magnetic field intensity, for both science and VFM calibration purposes. But it can also deliver experimental vector mode data, which are the data used here (see Léger et al. (2015), for more information on the ASM experimental vector mode). The payload also includes a dual frequency GPS receiver and instruments to measure plasma and electric field parameters as well as gravitational acceleration (see Floberghagen et al. (The Swarm mission - an overview one year after launch. Earth, Planets and Space, in preparation) for more details).

The production of our candidate 2015.0 provisional IGRF model involved several steps, all of which are detailed below ("Methods" section). We first produced adequate experimental ASM vector data from the so-called Level 0 files containing the basic vector mode output of the ASM instruments (in engineering units). These files were provided to us, following an agreement between ESA and the French space agency Centre National d'Etudes Spatiales (CNES), who funded and provided the ASMs as customer-furnished instruments. We next assembled a data set well suited to the purpose of building an IGRF candidate model. This involved using appropriate selection criteria. A very useful selection criterion we included was the requirement that whenever an ASM vector datum had been selected on a satellite, a synchronous nominal L1b datum (produced by ESA, based on the calibrated vector fluxgate magnetometer (VFM) instruments) also had to be available on the same satellite. As we shall later see, this (mild) criterion ensured the availability of synchronized ASM and L1b data sets for comparison purposes. The third step involved the production of a so-called parent model, best describing the magnetic field of internal origin over the 10-month time period covered by the selected data. This model consists of both a static field (described in terms of a spherical harmonic $(\mathrm{SH})$ representation up to degree and order 40) and a constant secular variation (SV, described up to degree and order 8). It was produced in a very similar way as a more advanced model (static field up to degree and order 45 , SV up to degree and order

\section{Flight direction $\rightarrow$}

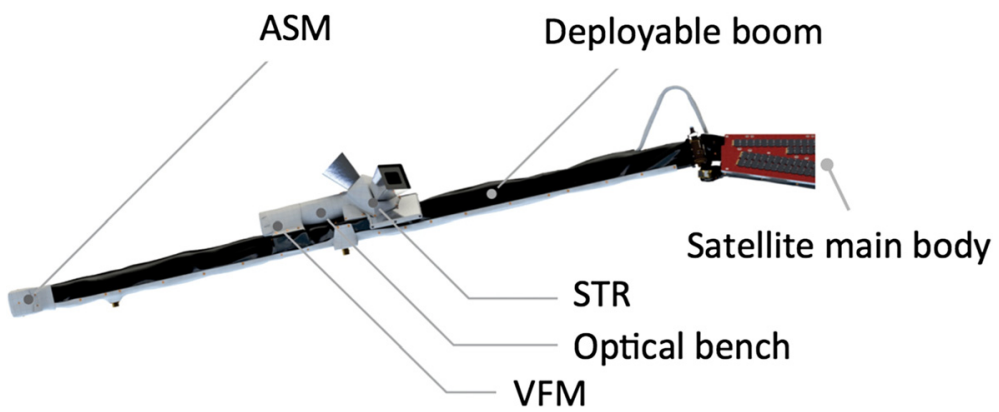

Fig. 1 Magnetometer payload setup. All instruments of the magnetometer payload are mounted on a deployable boom, with the vector field magnetometer (VFM) and the star tracker (STR) assembly sharing a common rigid optical bench and the absolute scalar magnetometer (ASM) mounted $2 \mathrm{~m}$ away, at the tip of the boom. The main body of the satellite (not shown) is on the right (towards the direction of flight, as indicated by the arrow) 
13) just published by Hulot et al. (2015b), also based on ASM vector data, but which could take advantage of more data (almost a year, not being limited by the October 1, 2014, delivery deadline which applied to IGRF candidate models). The final step simply consisted in extrapolating the parent model to epoch 2015.0, using the model's own SV and truncating it at degree and order 13 to produce our candidate 2015.0 provisional IGRF model.

The assessment of this model is discussed in the "Results and discussion" section below, relying on comparisons with some auxiliary models, which we built in exactly the same way but using our alternative L1b data set. We also discuss comparisons of our candidate model with the final IGRF model (Thébault et al. 2015a). Finally, we conclude this paper on the lessons learnt from building such an ASM-only IGRF candidate model ("Conclusions" section).

\section{Methods}

\section{Experimental ASM-V data}

The data that we used have been acquired using the ASM instruments on board the satellites of the Swarm mission. These instruments are absolute scalar magnetometers with a high-sampling rate $(1-\mathrm{kHz}$ internal sampling rate) equipped with three orthogonal sets of coils, each producing magnetic modulations with wellcontrolled amplitudes $(50 \mathrm{nT})$ and frequencies (adequately chosen within the $1-$ to $100-\mathrm{Hz}$ bandwidth) that add up to the natural field $\overrightarrow{B_{0}}(t)$. Analyzing the resulting scalar field $\left|\overrightarrow{B_{0}}(t)+\sum_{i=1}^{3} \overrightarrow{b_{i}} \cos \left(\omega_{i} t\right)\right|$ using real-time de-convolution is then what makes it possible for the instruments to deliver synchronized co-located, but independent, $1-\mathrm{Hz}$ vector and scalar readings (see Gravrand et al. (2001), Léger et al. (2009)). The key fact that vector and scalar readings are independent from each other is what allows the vector components to be calibrated for scaling factors, non-orthogonality, and possibly other parameters. The detailed procedure applied to calibrate the vector data used here, to which we will refer as the experimental ASM-V data, can be found in Léger et al. (2015).

In practice, the ASM-V data were derived on a daily basis from L0 files containing the necessary input for producing both the three ASM vector components (in the ASM frame of reference) and the independent ASM scalar data (the only ASM data otherwise used by ESA in the nominal processing of the L1b data). The processing starts with a self-calibration procedure, during which all calibration parameters are recovered by minimizing the residuals between the modulus of the reconstructed vector field and the independently measured ASM scalar value (see Gravrand et al. (2001), Léger et al. (2015)). The resulting calibration parameters are stored in a socalled characterization and calibration data base (CCDB) file. For the purpose of the present study, calibration was done on a daily basis, and the CCDB updated accordingly, using a 3-day sliding window scheme (parameters for the CCDB of a given day being optimized by minimizing residuals over the day of interest, the previous day, and the day after; see Léger et al. (2015)). This led to residuals with no bias (since the same sensor is used to infer both the scalar and vector components) and a noise level of $\sigma=2.3,2.4$, and $4.5 \mathrm{nT}$ for, respectively, Alpha, Bravo, and Charlie (one standard deviation, before any outlier rejection or data selection; see Léger et al. (2015)). Using these pseudo-daily CCDBs, calibrated vector data could thus be produced every second at $t_{\mathrm{ASM}}$ instrument's time stamps. However, these data (and the ASM scalar data) still had to be corrected for satellite stray fields. They also had to be synchronized to the $t_{\mathrm{UTC}}$ times used in all nominal L1b files (using a cubic B-spline interpolation method). The information needed for this was taken from nominal L1b files provided by ESA. Applying these corrections and synchronization then led to $1-\mathrm{Hz}$ calibrated and corrected ASM vector data in the ASM frame, together with ASM scalar data, fully synchronized with all official L1b data. These ASM-V vector data, provided in the ASM instrument frame, are formally analogous to the official L1b calibrated VFM data provided in the VFM instrument frame. They can be used in exactly the same formal way for modeling purposes. The ASM scalar data produced in the process can also be compared to the ASM scalar data stored in official L1b data files. Differences are very small (root mean square (rms) of $30 \mathrm{pT}$ ) and simply testify for the slightly different way strayfield corrections and synchronizations are implemented in the two (ours and ESA's) processing chains.

\section{Data selection}

Only data from the two Alpha and Bravo satellites were considered, the ASM-V data produced on Charlie being significantly noisier (recall above). This did not turn out to be a major limitation for the purpose of the present study, since Charlie is anyway orbiting very close to Alpha compared to the length scales of the main field to be modeled (note that having Charlie close to Alpha can nevertheless be taken advantage of for modeling the high-degree field, using the scalar ASM and nominal L1b vector data on Charlie, as illustrated in, e.g., Olsen et al. (2015)). The data selected extended from November 29, 2013 (1 week after launch) to September 25, 2014 (1 week before the deadline for the delivery of the IGRF candidate models). Some data were manually removed in an ad hoc manner, based on early inspection of the 
ASM-V data (early in the mission, to avoid using data at times of known or suspected issues either obviously or potentially affecting these data, such as satellite manoeuvres): between 27/01/2014 and 06/02/2014 (inclusive) for Alpha, and on 05/12/13 between 09:36 and 12:00, plus between 08/12/2013 and 17/12/2013 (inclusive) for Bravo. Only data from dark regions (sun at least $10^{\circ}$ below the horizon) were used since our modeling procedure did not involve modeling the magnetic fields produced by ionospheric currents (see below). The strength of the magnetospheric ring current, estimated using the ring current (RC) index (Olsen et al. 2014), was required to change by at most $2 \mathrm{nT} / \mathrm{h}$. Geomagnetic activity was required to be low, such that $\mathrm{Kp}<2+$. Merging electric field at the magnetopause was required not to be too large (details of how this criteria is defined are to be found in Olsen et al. (2014), section 2.1; suffice to say here that we used a more relaxed constraint than in CHAOS-4, based on averages over the past twelve 5-min values: $\left.E_{\mathrm{m}, 12}<3,3 \mathrm{mV} / \mathrm{m}\right)$. Finally, selection of scalar versus vector data was decided in the following way: for high quasi-dipole (QD) latitudes (higher than $55^{\circ}$ in each hemisphere), select only scalar ASM data to avoid signals from field-aligned currents (definition of QD latitudes are to be found in Richmond (1995)); for other latitudes, if the scalar residual (difference between the modulus of the ASM-V vector and the ASM scalar data) is less than $0.3 \mathrm{nT}$ and the ASM piezoelectric motor has not been activated within the previous $3 \mathrm{~s}$ (using ASM housekeeping information, also available), keep the ASM$\mathrm{V}$ data, else dismiss and use the ASM scalar data. The latter selection criterion is important, since piezoelectric motor activation is a cause of significant perturbation of the vector mode data. Implementing this criterion substantially reduces the noise level in the ASM-V data (by up to $30 \%$; see Léger et al. (2015)). Once collected, selected scalar and vector data were decimated by a factor of 60 and 8 to ensure that a similar weight is given to both data sets while keeping an adequate geographical sampling for the present purpose.

As mentioned in the "Background" section, to also be able to compute models based on L1b VFM data in exactly the same way as our candidate model based on ASM-V data (for comparison purposes), an additional mild selection criterion was added to all the above criteria: that for each ASM-V datum selected, a meaningful (i.e., not obviously inconsistent) official L1b datum was also available at exactly the same time on the same satellite, with vector field components within $500 \mathrm{nT}$ (and scalar field within $100 \mathrm{nT}$ ) of predictions from the CHAOS-4 model of Olsen et al. (2014), up to degree and order 20, linearly forwarded to the appropriate epoch (starting from epoch 2013.5 and using the model's SV computed at epoch 2013.5). Since this was implemented, two additional data sets could also be assembled.
One, which simply used nominal L1b VFM vector data in the VFM instrument frame (release 0302 when available, otherwise release 0301) in place of the ASM-V data; the other, built in the same way, except that each L1b VFM vector datum (still in the VFM instrument frame) was systematically normalized by the synchronous ASM scalar datum to ensure an exact match of its norm with this scalar datum. Both these data sets, to which we will refer as the VFM and normalized-VFM data sets, respectively, were thus constructed to include the same amount of data and be completely synchronous with our experimental ASM-V data set.

Figure 2 provides the altitude of the Alpha and Bravo satellites during the period modeled and the coverage of the selected data as a function of latitude and time. Note how critical the latest data were to avoid a dangerously short temporal coverage of the Northern latitudes (an issue entirely related to the dark region selection criteria and not to the ASM instrument itself). In all, we used $3 \times 65,169$ vector and 66,454 scalar data from the Alpha satellite and $3 \times 74,123$ vector and 67,095 scalar data from the Bravo satellite. This made a total of 551,425 data. This, we note, is about a factor 2 less than the nearly $10^{6}$ data used in the more advanced model recently produced by Hulot et al. (2015b), who could use data extending up to November 6, 2014, and selected in a slightly different way (taking advantage of the lessons learnt from constructing the models presented here).

\section{Parent model parameterization, estimation, and regularization}

The modeling procedure used to produce our parent model is similar to the one used by Hulot et al. (2015b) and Olsen et al. (2014), and we refer the reader to these publications for detailed equations and explanations. Here, we only provide a summary description of the method used and parameters inverted for.

The field was assumed to be potential, with both internal and external sources. Internal sources, which account for both the core and the lithospheric fields were represented by a spherical harmonic expansion up to degree and order 40 (at reference radius $a=6371.2 \mathrm{~km}$ ). A constant secular variation up to degree and order 8 was also used to model the core field evolution over the $25 / 11 / 2013$ to $25 / 11 / 2014$ modeled time interval. Parameters describing the internal part of the field thus consisted of $40 \times 42=1680$ static Gauss coefficients and $8 \times 10=80$ secular variation Gauss coefficients.

Signals from external sources were described as explained in Olsen et al. (2014) equations (4-5) and involved two different contributions. One described the signal from remote magnetospheric sources, modeled as a zonal external field of degree 2 in geocentric solar 

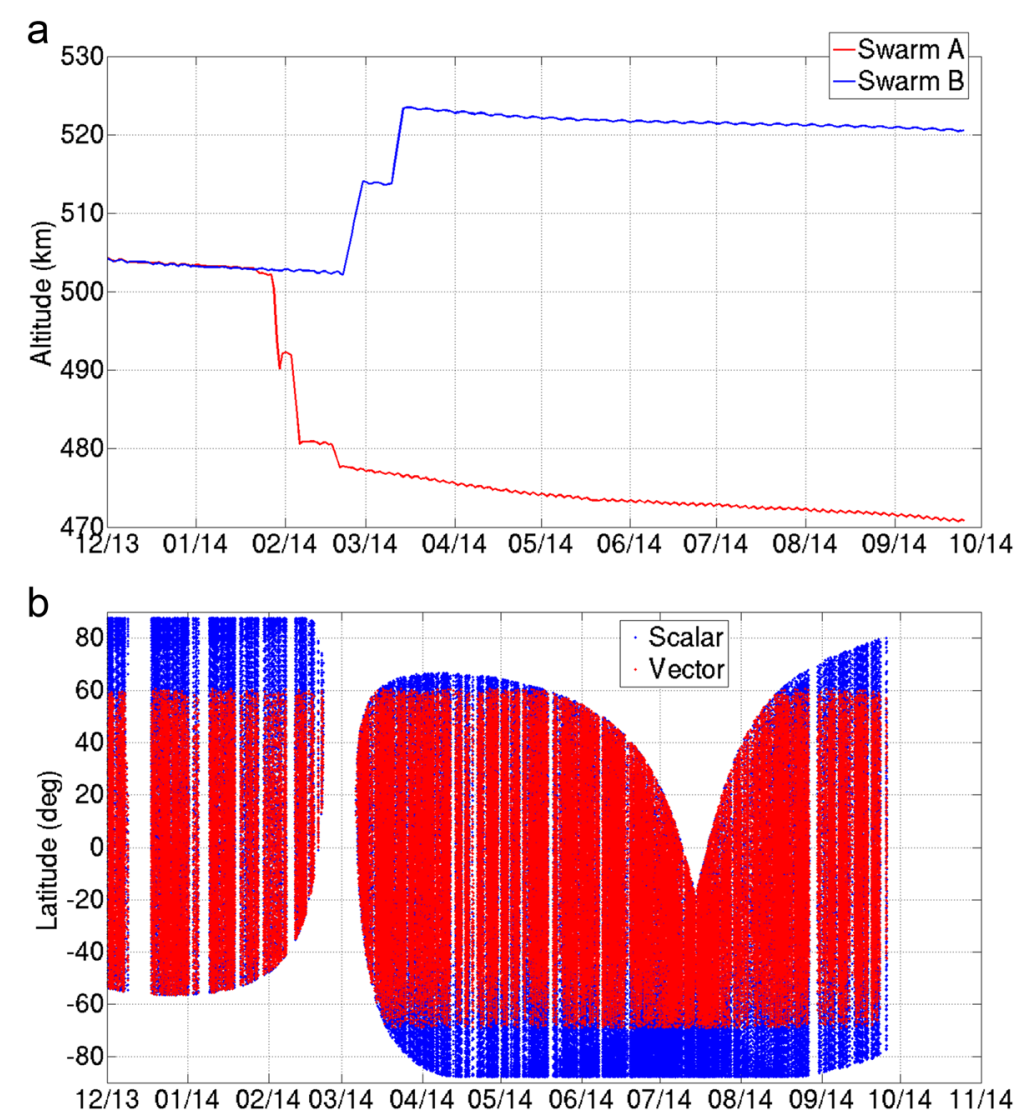

Fig. 2 Data geographical coverage. a Daily mean altitude of the Alpha (red) and Bravo satellites (blue); $\mathbf{b}$ geographic latitude coverage versus time for the vector (red) and scalar (blue) data satisfying the selection criteria and used in the models

magnetospheric (GSM) coordinates (for a definition of such coordinates, see, e.g., Hulot et al. (2015a)). The other described signals from the near-magnetospheric ring current in solar magnetic (SM) coordinates (see again Hulot et al. (2015a)). It assumed a static contribution, modeled up to degree and order 2 , but also relied on the so-called RC index (Olsen et al. 2014, computed independently from observatory data, prior to the model computation), three static regression factors, and a number of "RC baseline corrections" to also account for undetermined (by ground magnetic observations) absolute baseline of the RC signal. Referring to the notations of Olsen et al. (2014), equations (4-5), the parameters we used for the external field, thus were (13 in total) as follows: $q_{1}^{0, \text { GSM }}$, $q_{2}^{0,}$ GSM for the remote magnetospheric sources (two coefficients); $q_{2}^{m}, s_{2}^{m}$ for the static degree 2 component of the ring current (five coefficients); $\hat{q}_{1}^{0}, \hat{q}_{1}^{1}, \hat{s}_{1}^{1}$ for the regression factors (three coefficients); $\Delta q_{1}^{0}, \Delta q_{1}^{1}, \Delta \mathrm{s}_{1}^{1}$ for the baseline corrections (three coefficients; note that contrary to what was done in Olsen et al. (2014) and Hulot et al. (2015b), only static baseline corrections were used here).

Euler angles describing the rotation between the ASM-V frame and the STR frame were also solved for by bins of 10 days (introducing an additional $3 \times 30$ parameters per satellite, hence 180 in total). The total number of parameters to be estimated thus amounted to 1680 (static Gauss coefficients) + 80 (secular variation Gauss coefficients) +13 (external field coefficients) + 180 (Euler angles) $=1953$ parameters.

These 1953 model parameters were estimated from the 551,425 data, using an iteratively reweighted leastsquares algorithm with Huber weights. The cost function to minimize was $e^{T} C^{-1} e$, where $e=d_{\mathrm{obs}}-d_{\bmod }$ is the difference between the vector of observations $d_{\text {obs }}$ and the vector of model predictions $d_{\text {mod }}$, and $C$ is the data covariance matrix. Note, in particular, that no regularization was applied. A $\sin (\theta)$ weight was introduced (where $\theta$ is the geographic co-latitude), to balance the geographical sampling of data. In all computations, $a$ priori data error variances were otherwise set to $2.5 \mathrm{nT}$ for both scalar and vector data, also assuming an isotropic attitude error of 10 arcsecs (using the formalism of Holme and Bloxham (1996)). Due to both scalar data and Huber weights being involved, the cost function depends nonlinearly on the model parameters, and the solution was obtained iteratively, using a Newton-type 
algorithm. The (static) starting model was CHAOS-4 (with no Swarm data) up to degree and order 13, linearly forwarded to epoch December 1, 2013 (starting from epoch 2013.5 and using the model's SV computed at epoch 2013.5). The final model was obtained after ten iterations to check convergence (misfits did not change by more than $0.01 \mathrm{nT}$ between the two last iterations). The sensitivity to the starting model was also checked, and the final model had converged to virtually the same Gauss coefficients (well within the estimated errors we later provide). Since this final model was computed without involving any regularization, it formally represents the best linear evolution of the field of internal origin (static for degrees larger than 8) over the $25 / 11 / 2013$ to $25 / 11 / 2014$ time interval, given the constraints provided by the data available between $29 / 11 / 2013$ and $25 / 09 / 14$. It is not formally anchored to any particular epoch but may be viewed as best representing the field at the central epoch of the data coverage (end of May 2014).

\section{Derivation of the ASM-V 2015.0 IGRF candidate model and of auxiliary models}

Applying the methodology described above to the selected ASM-V data led to the production of an ASM-V parent model. This parent model was next forwarded to epoch 2015.0, using its own secular variation (up to degree and order 8 ) and truncated at degree and order 13. This led to the ASM-V 2015.0 IGRF candidate model, which we delivered on October 1, 2014, as requested by the call.

Useful auxiliary information was also provided to the task force in the form of additional files. This extra information included the Gauss coefficients of the SV model associated with the ASM-V parent model, which could have been proposed as a candidate IGRF SV model for the interval 2015.0-2020.0 but was eventually provided as an ASM-V 2015-2020 SV test model. Indeed, rules of the call for candidate models requested leading institutions to propose no more than one candidate for the same IGRF type of model, and it turned out that IPGP was also leader in the production of another predictive IGRF secular variation candidate model (using a data assimilation type of strategy, Fournier et al. (2015); see also Fournier et al. (2010)).

For both the ASM-V 2015.0 IGRF candidate and ASM-V 2015-2020 SV test models, simple uncertainty estimates were also provided. These were computed by just splitting the original data set into two sub-data sets. Numbering days from first to last day of available data, all odd number days were put in one sub-data set, all even days in the second one. We checked that this indeed leads to very similar data distributions in both sub-data sets. These sub-data sets were then used to compute two sub-models using exactly the same procedure and parameters as when using the full data set. These two sub-models (indexes 1 and 2, using index 0 to refer to the original model) were then used to infer a rough $\sigma$ estimate for each Gauss coefficient in the following way:

$$
\sigma\left(g_{n}^{m}\right)=\sqrt{1 / 2\left(\left(g_{n, 1}^{m}-g_{n, 0}^{m}\right)^{2}+\left(g_{n, 2}^{m}-g_{n, 0}^{m}\right)^{2}\right)}
$$

and likewise for the $h_{n}^{m}$ coefficients and SV Gauss coefficients.

A number of useful auxiliary models were also built and provided. For this, we used the L1b VFM and normalized-VFM data sets in place of the ASM-V data set but otherwise relied on the same modeling procedure (using exactly the same choice of parameters). Models built in this way from the VFM data set are later referred to as the VFM parent, VFM 2015.0, and VFM 2015-2020 SV auxiliary models (analogous to the ASM-V parent, ASM-V 2015.0 IGRF candidate, and ASM-V 2015-2020 SV test models, respectively), while those built from the normalized-VFM data set are later referred to as the N-VFM parent, N-VFM 2015.0, and N-VFM 2015-2020 SV auxiliary models.

\section{Results and discussion}

\section{Misfit statistics and error estimates}

Residual statistics of the ASM-V parent model are provided in Table 1. As can be seen, scalar residuals are larger at high latitudes $(4.81 \mathrm{nT})$ than at low latitudes $(2.30$ $\mathrm{nT}$ ). Similar effects have been observed in pre-Swarm era models, such as CHAOS-4 (Olsen et al. 2014), reflecting the larger contribution of un-modeled sources at high latitudes. Considering the rather simplified modeling procedure used here (recall we only solve for 1953 parameters), these misfits are of reasonable magnitudes. Also worth noting is the fact that the scalar misfits for the ASM-V parent model are nearly identical to those obtained for the VFM and N-VFM parent models (also provided in Table 1). At high latitudes, this reflects the fact that all three models rely on the same ASM scalar data. At lower latitudes, however, this result is less obvious, since the models are also constrained by their respective ASM-V or VFM vector data.

The impact of using ASM-V vector data (rather than VFM vector data) at low latitudes is more clearly found in the geocentric components $B_{n} B_{\theta}$, and $B_{\phi}$. Indeed, the residuals of the ASM-V parent model with respect to these components are systematically larger than those of the VFM and N-VFM parent model. The increase, however, is quite small (by 12 to $34 \%$ depending on the misfit, the largest increase being observed on $B_{r}$ ), 
Table 1 Number $N$ of data points and Huber-weighted mean and rms misfits (in nT) for the ASM-V, VFM, and N-VFM parent models

\begin{tabular}{|c|c|c|c|c|c|c|c|c|}
\hline & & \multirow[t]{2}{*}{$N$} & \multicolumn{2}{|l|}{ ASM-V } & \multicolumn{2}{|l|}{ VFM } & \multicolumn{2}{|l|}{ N-VFM } \\
\hline & & & mean & $\overline{\mathrm{rms}}$ & mean & $\overline{r m s}$ & mean & $\mathrm{rms}$ \\
\hline Alpha + & $F_{p}$ & 49,214 & -0.27 & 4.81 & +0.00 & 4.80 & -0.01 & 4.80 \\
\hline \multirow[t]{4}{*}{ Bravo } & $F_{\mathrm{NP}}+B_{B}$ & 223,627 & +0.04 & 2.30 & +0.02 & 2.27 & +0.01 & 2.27 \\
\hline & $B_{r}$ & 139,292 & -0.02 & 2.45 & -0.06 & 1.76 & -0.02 & 1.83 \\
\hline & $B_{\theta}$ & 139,292 & -0.03 & 3.56 & +0.06 & 3.18 & +0.06 & 3.17 \\
\hline & $B_{\varphi}$ & 139,292 & -0.16 & 2.96 & -0.13 & 2.61 & -0.15 & 2.60 \\
\hline \multirow[t]{5}{*}{ Alpha } & $F_{p}$ & 24,294 & -0.31 & 4.94 & -0.05 & 4.93 & -0.06 & 4.93 \\
\hline & $F_{\mathrm{NP}}+B_{B}$ & 107,329 & +0.08 & 2.32 & -0.03 & 2.29 & +0.06 & 2.29 \\
\hline & $B_{r}$ & 65,169 & +0.00 & 2.47 & -0.05 & 1.77 & +0.01 & 1.83 \\
\hline & $B_{\theta}$ & 65,169 & -0.13 & 3.61 & +0.14 & 3.19 & +0.04 & 3.19 \\
\hline & $B_{\varphi}$ & 65,169 & -0.19 & 3.02 & -0.15 & 2.62 & -0.17 & 2.61 \\
\hline \multirow[t]{5}{*}{ Bravo } & $F_{p}$ & 24,920 & -0.23 & 4.68 & +0.05 & 4.67 & +0.04 & 4.67 \\
\hline & $F_{\mathrm{NP}}+B_{B}$ & 116,298 & -0.01 & 2.28 & +0.06 & 2.26 & -0.03 & 2.25 \\
\hline & $B_{r}$ & 74,123 & -0.04 & 2.44 & -0.07 & 1.74 & -0.05 & 1.83 \\
\hline & $B_{\theta}$ & 74,123 & +0.06 & 3.51 & -0.01 & 3.18 & +0.08 & 3.16 \\
\hline & $B_{\varphi}$ & 74,123 & -0.14 & 2.91 & -0.12 & 2.60 & -0.14 & 2.60 \\
\hline
\end{tabular}

$F_{P}$ refers to scalar data with (absolute) quasi-dipole latitude larger than $55^{\circ}$ (recall that no vector data are selected at such latitudes); $F_{\mathrm{NP}}$ refers to scalar data at other latitudes (selected whenever vector data are not selected); $B_{B}$ refers to the vector component along the main field direction; $B_{r}, B_{\theta}$ and $B_{\varphi}$ refer to the geocentric components

considering that the noise of the $1-\mathrm{Hz}$ ASM-V vector components is less than $2 \mathrm{nT}$ rms (see Léger et al. (2015)) and that the ASM is sitting on a boom much further away from the STR than the VFM, in less stable mechanical conditions (recall Fig. 1 and see Fratter et al. (Swarm absolute scalar magnetometers first in - orbit results. Astronautica, Acta, in review)). Given the "scalar residual" issue known to have affected the VFM L1b data available at the time of this study (testifying for perturbations affecting these data at the level of possibly $2 \mathrm{nT}$ or even slightly more, see Floberghagen et al. (The Swarm mission - an overview one year after launch. Earth, Planets and Space, in preparation)), this was an encouraging sign that the ASM-V 2015.0 IGRF candidate could indeed be proposed as a useful independent candidate model for IGRF-2015.

To assess how much uncertainty affects our ASM-V 2015.0 IGRF candidate model, one can first refer to the uncertainties $\sigma\left(g_{n}^{m}\right)$ and $\sigma\left(h_{n}^{m}\right)$ computed from Eq. 1 (and provided with the model). To get a better grasp of the geographical distribution of the errors, however, one can also plot a rough estimate of the error affecting the radial component $B_{r}(\theta, \phi)$ predicted by the model at the Earth's surface, computed in the following way:

$$
\sigma\left(B_{r}(\theta, \phi)\right)=\sqrt{1 / 2\left(\left(B_{r, 1}(\theta, \phi)-B_{r, 0}(\theta, \phi)\right)^{2}+\left(B_{r, 2}(\theta, \phi)-B_{r, 0}(\theta, \phi)\right)^{2}\right)}
$$

where, as in Eq. 1, indexes 1 and 2 refer to the two sub-models computed from the two ASM-V sub-data sets, and index 0 refers to the original ASM-V candidate model.

Figure 3 shows a map of this quantity. It highlights two important facts: first, that regions where the model suffers the largest errors are located at high northern (roughly centered on the north geomagnetic pole over Greenland, between 4 and $12 \mathrm{nT}$ ) and southern latitudes (up to $4 \mathrm{nT}$ ), and second, that errors elsewhere are limited to much lesser values (typically 1 to $2 \mathrm{nT}$ ). The large Greenland "error spot" simply reflects the fact that high northern latitudes suffered from nighttime Swarm data being available only early in the mission (until February 2014, the most recent data failing to again reach northern latitudes above $80^{\circ}$, recall Fig. $2 \mathrm{~b}$ ). This led the associated ASM-V 2015-2020 SV test model to be less constrained at these latitudes (as could indeed be checked by plotting a figure analogous to Fig. 3, not shown). This, combined with the fact that, as a general rule, ionospheric signals not accounted for by our models are strongest at high-magnetic latitudes, resulted in larger errors being propagated to epoch 2015.0 in this high-magnetic latitude region when building the ASM-V 2015.0 IGRF candidate. High southern latitudes suffered from the opposite issue of not having been sampled early in the mission (not until April 2014, see again Fig. 2b). But the later data coverage (until the beginning of September 2014) turned out to both be long enough and close enough to epoch 2015.0 to not lead to as big an issue. Finally, it should be noted that a very similar geographical distribution of errors has been found to also affect both the VFM and N-VFM 2015.0 auxiliary models, confirming that the issue is mainly related to the spatiotemporal distribution of the data. This first assessment thus points at the key limitation of our ASM-V IGRF 2015.0 candidate model (and of the ASM-V 2015-2020 SV test model) being related to the unfortunate circumstances that IGRF candidate models had to be released for October 1, 2014, just 1 month short of Swarm being able to again acquire high northern nighttime data.

\section{Comparing the ASM-V candidate model with auxiliary and IGRF official models}

Important additional insight can be gained from intercomparisons of the ASM-V 2015.0 IGRF candidate 


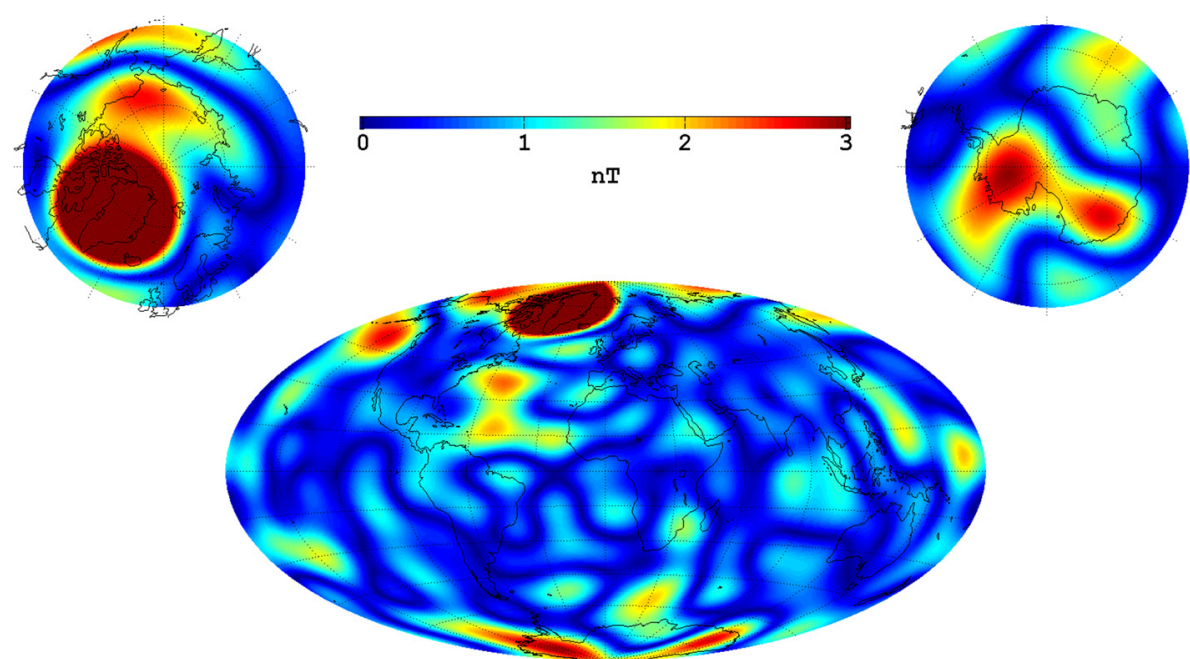

Fig. 3 Error estimates on the field predicted by the ASM-V IGRF 2015.0 candidate model. Plotted is a map of the estimated local error on the $B_{r}$ component predicted by the ASM-V IGRF 2015.0 candidate model at the Earth's surface (i.e., at reference radius $a=6371.2 \mathrm{~km}$ ), as estimated from $\sigma\left(B_{r}(\theta, \varphi)\right)$ (Eq. 2). Note that the scale is deliberately limited to $3 \mathrm{nT}$ (maximum values are reached over Greenland, the absolute value being $12 \mathrm{nT})$

model, the two VFM and N-VFM 2015.0 auxiliary models, and the official 2015.0 IGRF model (now published, see Thébault et al. (2015a)). Figure 4 shows relevant Lowes-Mauersberger spectra (Mauersberger 1956, Lowes 1966). As can be seen, the two VFM and NVFM 2015.0 auxiliary models appear to be in extremely good agreement, differences being well below the $10^{-1}$ $\mathrm{nT}^{2}$ level in spectral terms. This amounts to a rms difference at the 0.4-nT level at the Earth's surface (cumulated up to degree 13). Clearly, the impact of renormalizing VFM vector data using ASM scalar data is very small. This contrasts with the impact of using the directional information from the ASM-V data rather than that from the VFM data. Indeed, Fig. 4 shows that the agreement between the ASM-V 2015.0 IGRF candidate model and the VFM 2015.0 auxiliary model is now more in the 1- $\mathrm{nT}^{2}$ range, translating into a rms difference of $3.6 \mathrm{nT}$. Encouragingly, however, these spectral differences remain comparable to the spectral differences a posteriori observed between either of these models and the official 2015.0 IGRF model (also shown in Fig. 4).

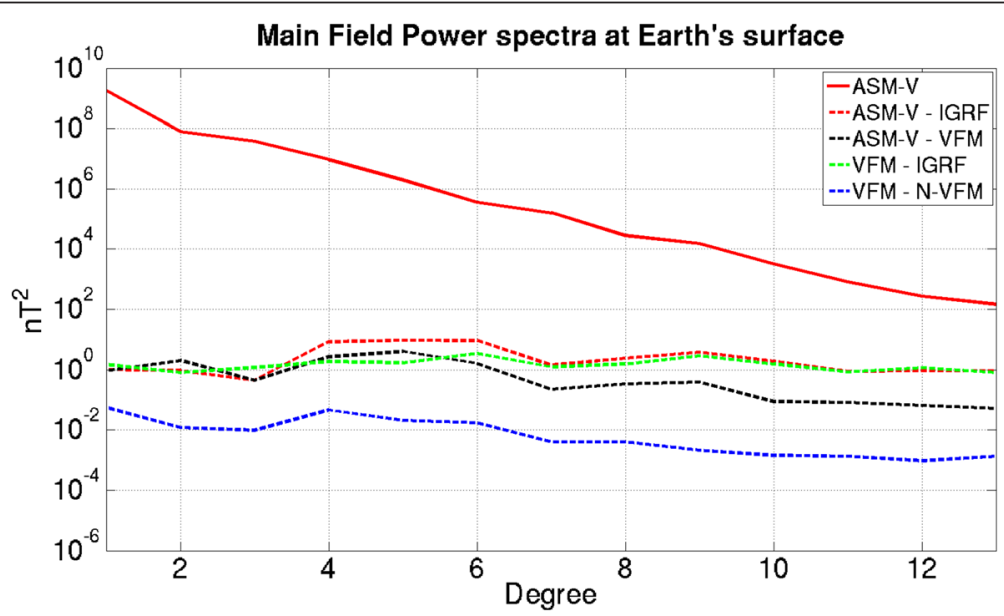

Fig. 4 Spectral comparison of the ASM-V 2015.0 IGRF candidate model with other models. Lowes-Mauersberger spectra of the ASM-V 2015.0 IGRF candidate model (solid red), as well as of the differences between this model and, respectively, the official 2015.0 IGRF model (dashed red) and VFM 2015.0 auxiliary model (dashed black). Also shown, the spectra of the differences between the VFM 2015.0 auxiliary model and, respectively, the official 2015.0 IGRF model (dashed green) and N-VFM 2015.0 auxiliary model (dashed blue); all at Earth's surface (i.e., at reference radius $a=6371.2 \mathrm{~km})$ 
Plotting the difference in the $B_{r}$ component predicted by the ASM-V 2015.0 IGRF candidate and VFM 2015.0 auxiliary models at the Earth's surface brings useful additional insight (Fig. 5a). It reveals that the highest discrepancies between the two models are to be found at high northern latitudes (up to nearly $20 \mathrm{nT}$ ), the agreement being much better elsewhere (within the 3-nT range, with another weak maximum at high southern latitude). This is very similar to what had been observed in the plot of error estimates shown in Fig. 3 and again suggests that the main cause of the enhanced high northern latitude differences is the lack of high northern latitude being available towards the end of September. Note, however, that the impact is now more significant and affecting a wider region centered on the north geographic pole rather than on the north geomagnetic pole. This reflects differences in the error revealed when relying on different, but synchronous, vector data sets (ASM-V versus VFM) rather than on a homogeneous data set split into two non-synchronous sub-data sets. While the former case mainly reflects the impact of differences in the readings of the ASM and VFM instruments for exactly the same spatiotemporal distribution of data, the latter mainly reflects differences due to highmagnetic latitude non-modeled ionospheric signals when relying on non-synchronous sub-data sets. Interestingly, plotting the analogous difference in the $B_{r}$ component predicted by the VFM 2015.0 and N-VFM 2015.0 auxiliary model reveals a much weaker effect (Fig. 5b), showing that it is again the mismatch in the directional information provided by ASM-V and VFM data sets that is mainly responsible for the enhancement of this highlatitude effect.

As a matter of fact, Figs. 3 and 5a, b nicely summarize the uncertainties likely affecting our ASM-V IGRF 2015.0 candidate model. In general (i.e., except at high latitudes), the noise level in the ASM-V data is responsible for uncertainties within the range of $2 \mathrm{nT}$ (Fig. 3); disagreements between the modulus of the VFM data and the ASM scalar data (the so-called "scalar anomaly", see Floberghagen et al. (The Swarm mission - an overview one year after launch. Earth, Planets and Space, in preparation)) are responsible for uncertainties within the range of $1 \mathrm{nT}$ (Fig. 5b); while, in contrast, disagreements between the directions of the VFM and ASM-V data are responsible for the largest uncertainties, within the range of $3 \mathrm{nT}$ (Fig. 5a). Interestingly, this is exactly the magnitude of the "vector anomaly" one can find when comparing the ASM-V and Level 1b VFM data at satellite level (not shown). This "vector anomaly" has a significant

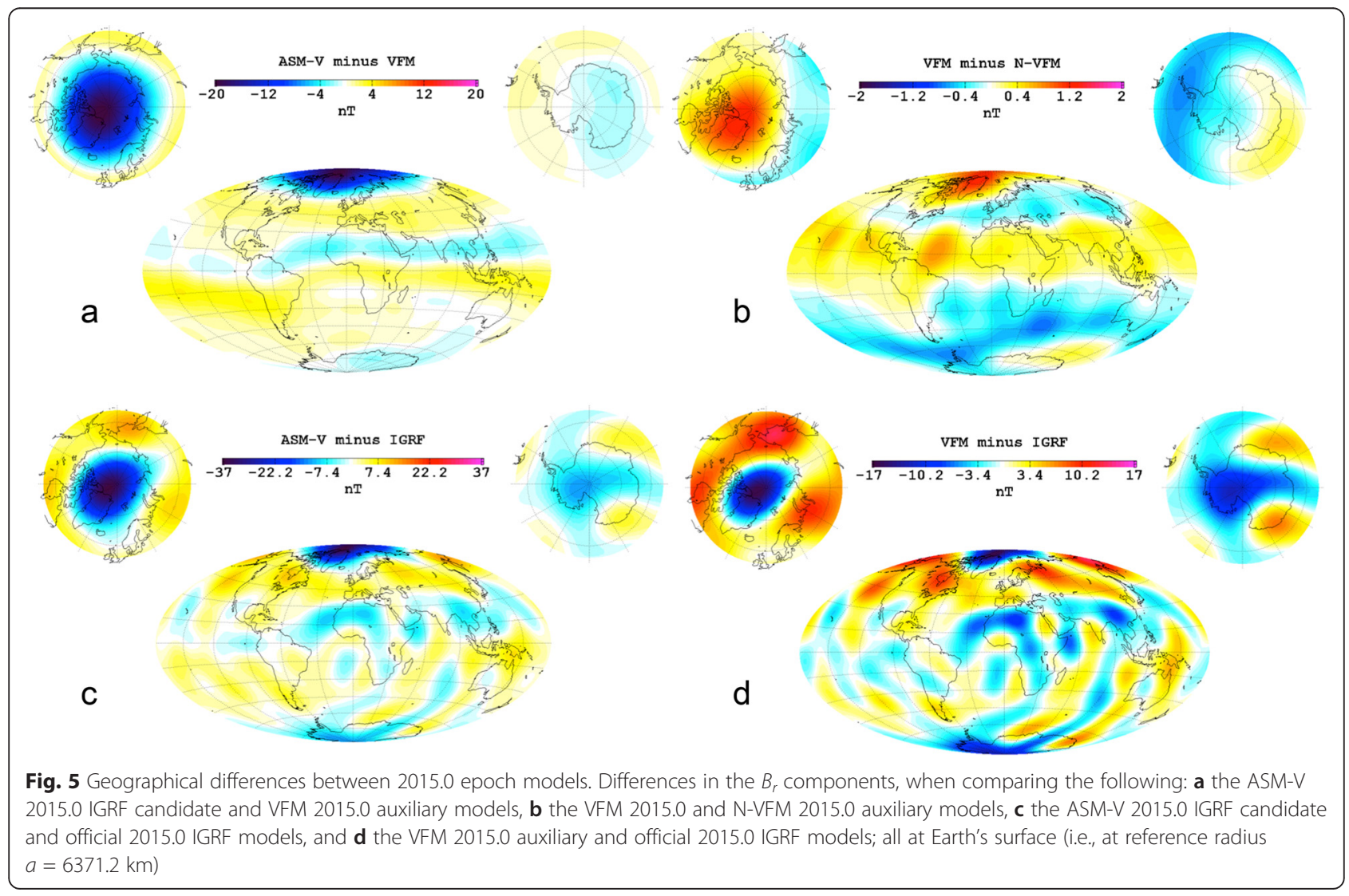


orbital component, including on the night side, and can be interpreted in terms of likely boom oscillations (though we note that this anomaly will inevitably also partly reflect the vector signature of what causes the previously mentioned scalar anomaly and may also have other, yet unidentified, causes). In any case, it is quite clear that the zonal pattern following the magnetic equator to be seen in Fig. 5a directly testifies for the way these anomalies mapped into the models. These various uncertainties, unfortunately, are amplified at the high northern latitudes, all in the same way, for the reasons already outlined.

Bearing all the above results in mind, we now turn to geographical comparisons of our ASM-V 2015.0 IGRF candidate model with the official 2015.0 IGRF model. We again plot the difference in the $B_{r}$ component predicted by these two models (Fig. 5c). For useful comparison purposes, we also plot the difference in the $B_{r}$ component predicted by our VFM 2015.0 auxiliary model and the official 2015.0 IGRF model (Fig. 5d). Consistent with the analysis carried out so far, the strongest disagreements between the candidate and official 2015.0 IGRF models occur at high northern latitudes with a maximum reaching $37 \mathrm{nT}$. This is almost the double of the analogous disagreement found in comparing the ASM-V 2015.0 IGRF candidate and VFM 2015.0 auxiliary models (Fig. 5a) and reflects the fact that the VFM 2015.0 auxiliary model itself also displays significant high northern latitude disagreements with the official 2015.0 IGRF model (Fig. 5d). Note, indeed, that by construction, differences plotted in Fig. $5 \mathrm{a}$, d add up to make the difference plotted in Fig. 5c. At other latitudes, however, differences between the candidate and official 2015.0 IGRF models are much weaker, peaking at $12 \mathrm{nT}$, and are very similar to the differences found between the VFM 2015.0 auxiliary model and the official 2015.0 IGRF model (peaking at $10 \mathrm{nT}$ ). This is very encouraging as it shows that the only significant impact of our decision to rely on ASM-V data rather than nominal L1b VFM data to produce our candidate model is in the form of disagreements limited to high northern latitudes, these disagreements being related to our modeling choice of only relying on Swarm data with an intrinsically limited spatiotemporal coverage.

We now turn to similar inter-comparisons of the ASM-V 2015-2020 SV test model, the two VFM and NVFM 2015-2020 SV auxiliary models, and the official 2015-2020 SV IGRF models. Figure 6, analogous to Fig. 4, shows that the two VFM and N-VFM SV auxiliary models are in extremely good agreement, well below the $10^{-1}(\mathrm{nT} / \mathrm{yr})^{2}$ level in spectral terms. This amounts to rms differences at the $0.5-\mathrm{nT} / \mathrm{yr}$ level at the Earth's surface (cumulated up to degree 8). It shows again that the impact of re-normalizing VFM vector data using ASM scalar data is very small. As one could have expected, the agreement between the ASM-V 2015-2020 SV test model and VFM 2015-2020 SV auxiliary model is not as good, though mostly below the $3(\mathrm{nT} / \mathrm{yr})^{2}$ level in spectral terms, translating into rms differences at the 3-nT/ yr level. Encouragingly, however, these spectral differences again remain smaller than the differences a posteriori observed between either of these models and the official 2015-2020 SV IGRF model (also shown in Fig. 6).

Finally, Fig. 7 confirms that most of the disagreements found between our ASM-V 2015.0 IGRF candidate model and all other 2015.0 models discussed here, including the official 2015.0 IGRF model, originate from disagreements between the 2015-2020 SV models used to reach epoch 2015. Note, indeed, the remarkable similarity between Figs. 5 and 7 (bearing in mind that all SV models are of maximum degree and order 8 ). This similarity validates our interpretation of the largest disagreements at the high northern latitudes being caused by similar differences being produced in the SV models as a result of the limited spatiotemporal distribution of the data set used. This limited distribution, combined with the fact that the SV models only extend to degree and order 8 , turns out to be responsible for the propagation at high latitudes of the disagreements between the ASM-V and VFM data used at mid-latitudes, despite the fact that the ASM-V, VFM, and N-VFM models otherwise rely on the same high-latitude scalar data. Finally, Fig. 7a also brings an interesting piece of information. Comparing it with Fig. 5a reveals that it displays far less of the distinct zonal pattern found along the magnetic equator in the difference between the ASM-V and VFM 2015.0 models, which we previously noted could be attributed to systematic "vector anomalies" between the ASM and VFM instruments. This is a clear indication that this vector anomaly indeed has a strong systematic pattern at all times.

\section{Conclusions}

Using ASM-V experimental vector data acquired on board the Swarm satellites, a very valuable 2015.0 IGRF candidate model, and an equally valuable 2015-2020 SV test model, could be derived. Analyzing errors possibly affecting these models nevertheless revealed some limitations. These limitations were shown to mainly be due to the restricted spatiotemporal distribution of the Swarm data available to produce the models on time for delivery to the task force. As a matter of fact, further evidence that time restriction was indeed the main issue can also be found in the fact that the higher resolution (up to degree and order 45, with a SV up to degree and order 13) model derived from the same ASM-V data in a very similar way by Hulot et al. (2015b), but with just a 


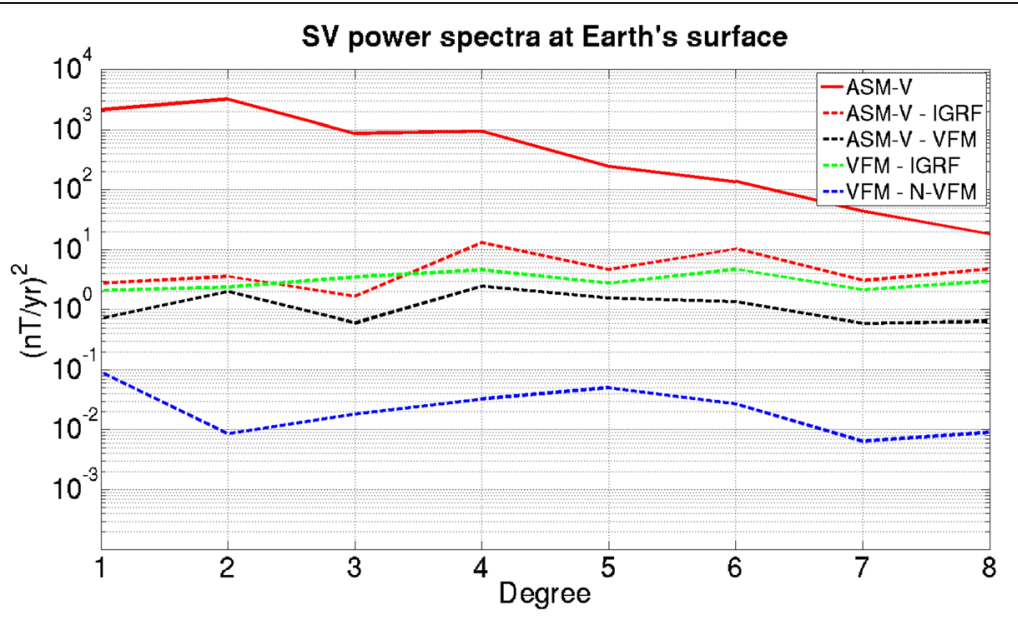

Fig. 6 Spectral comparison of the ASM-V 2015-2020 SV test model with other SV models. Lowes-Mauersberger spectra of the ASM-V 2015-2020 SV test model (solid red), as well as of the differences between this model and, respectively, the official 2015-2020 SV IGRF model (dashed red) and VFM 2015-2020 SV auxiliary model (dashed black). Also shown, the spectra of the differences between the VFM 2015-2020 SV auxiliary model and, respectively, the official 2015-2020 SV IGRF model (dashed green) and N-VFM 2015-2020 SV auxiliary model (dashed blue); all at Earth's surface (i.e., at reference radius $a=6371.2 \mathrm{~km}$ )

month and a half more ASM-V data, no longer is plagued by as strong limitations.

Comparing our candidate 2015.0 and 2015-2020 SV test models with analogous models based on the mission's nominal L1b VFM data showed that the greatest discrepancies were to be found at high northern latitudes. Not surprisingly, these are also the latitudes were our models displayed the greatest disagreement with the final official 2015.0 and 2015-2020 SV IGRF models. At other latitudes, however, the agreement was much better,

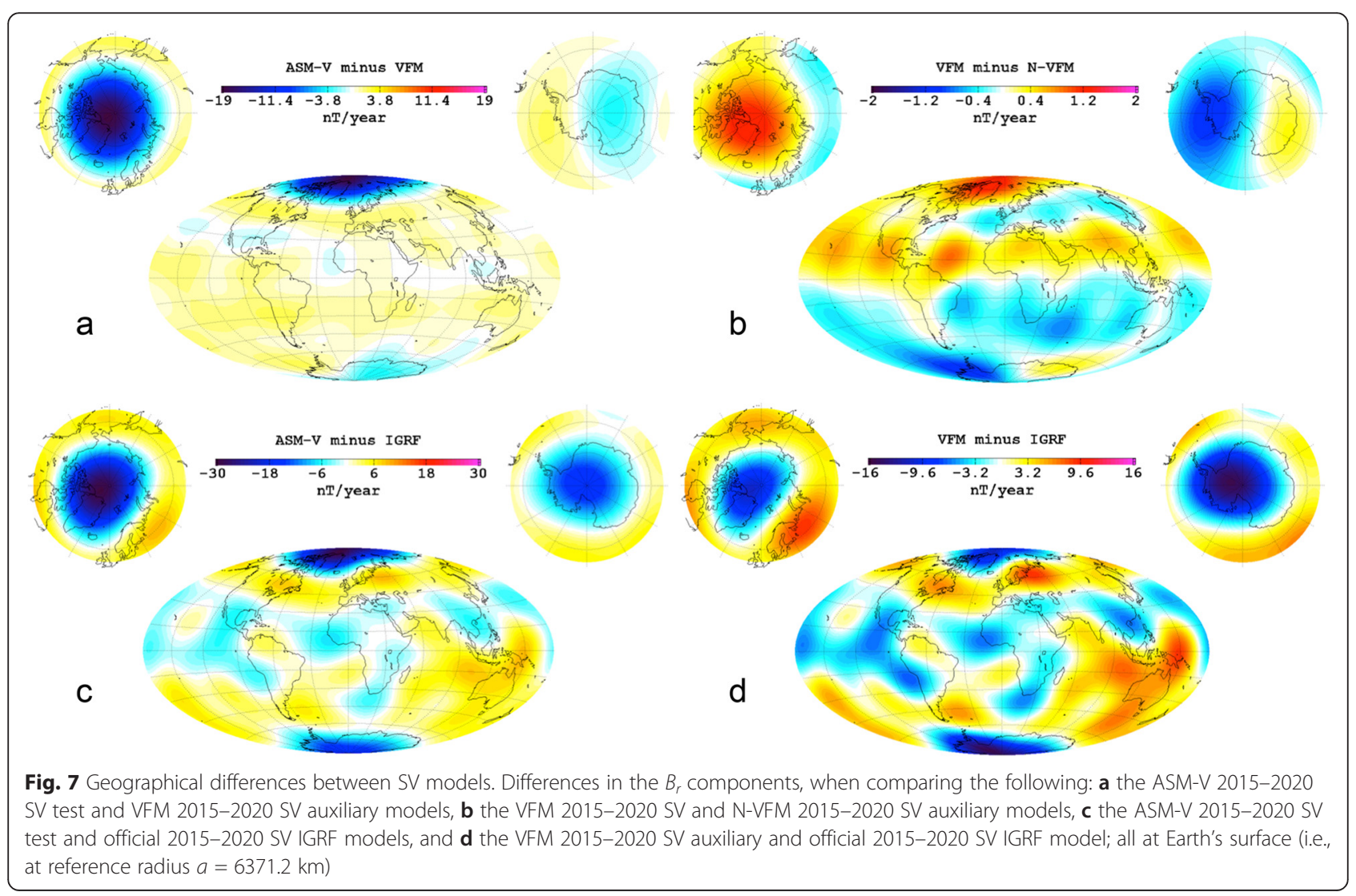


in fact very comparable to the type of disagreements found in most of the IGRF candidate models (see Thébault et al. (2015b)). This is quite remarkable, given that all other IGRF candidates heavily relied on the nominal L1b data of the Swarm mission or on ground data and that our model was the only one entirely and only relying on ASM-V data. These very nice performances led our ASM-V 2015.0 IGRF candidate model to contribute as one of the models finally used to build the official 2015.0 IGRF model, its contribution at high northern latitudes having been down-weighted by the weighing scheme decided by the task force (see Thébault et al. (2015b)).

This study thus showed that despite the ASM instruments being in a not-so-favorable mechanical position for vector field attitude restitution at the tip of the satellite's boom ( $2 \mathrm{~m}$ away from the optical bench common to the STR and VFM), its experimental vector mode could nicely be taken advantage of to build a very valuable 2015.0 IGRF field candidate. This was the first science achievement of these instruments, the first absolute scalar magnetometer capable of using the same sensor to also deliver absolute vector measurements on board satellites.

\section{Competing interests}

The authors declare that they have no competing interests.

\section{Authors' contributions}

PV carried out the model computations and participated in all steps of the study; GH, PI of the ASM instruments, designed the study, wrote the manuscript, and participated in all steps of the study; NO provided the CHAOS-4 sources of the inverse modeling software and participated in the model computation and interpretation; JML led the design of the ASM instruments and its processing chain at instrument level together with TJ and $F B ; \mathrm{LB}$ contributed to the processing chain and together with $\mathrm{PC}, \mathrm{XL}$, and $A C$ contributed to the quality analysis of the ASM vector mode data; $\mathrm{OS}$ and $\mathrm{AB}$ contributed to the management and production of the data; IF Swarm project manager for CNES, followed the entire study and contributed to the analysis of the vector mode data. All authors read and approved the final manuscript.

\section{Acknowledgements \\ The authors thank Chris Finlay for kindly providing the RC index needed for this study and two anonymous reviewers for constructive comments. They also thank the ESA Swarm project team for their collaboration in making ASM-V experimental data acquisition possible. They finally gratefully acknow- ledge support from the Centre National d'Etudes Spatiales (CNES) within the context of the "Travaux préparatoires et exploitation de la mission SWARM" project and from the European Space Agency (ESA) through ESTEC contract number 4000109587/13/I-NB "SWARM ESL". All Swarm L1b data are freely available from ESA at http://earth.esa.int/swarm. Experimental ASM-V data used for the present study are available from the corresponding author, subject to approval by CNES and CEA-Léti. All model coefficients can be downloaded from http://geomag.ipgp.fr/download/IPGP_IGR- F_ASMV.tar.gz. This is IPGP contribution $\mathrm{n}^{\circ} 3641$.}

\section{Author details}

'Institut de Physique du Globe de Paris, Sorbonne Paris Cité, Université Paris Diderot, CNRS, F-75005 Paris, France. ${ }^{2}$ DTU Space, National Space Institute, Technical University of Denmark, Kongens Lyngby, Denmark. ${ }^{3}$ CEA, LETI, MINATEC Campus, F-38054 Grenoble, France. ${ }^{4}$ Cooperative Institute for Research in Environmental Sciences, University of Colorado and National Geophysical Data Center, Boulder, CO, USA. ${ }^{5}$ Centre National d'Etudes Spatiales, Toulouse, France.
Received: 19 February 2015 Accepted: 5 June 2015

Published online: 20 June 2015

\section{References}

Finlay CC, Maus S, Beggan CD, Bondar TN, Chambodut A, Chernova TA, Chulliat A, Golovkov VP, Hamilton B, Hamoudi M, Holme R, Hulot G, Kuang W, Langlais B, Lesur V, Lowes FJ, Lühr H, Macmillan S, Mandea M, McLean S, Manoj C, Menvielle M, Michaelis I, Olsen N, Rauberg J, Rother M, Sabaka TJ, Tangborn A, Tøffner-Clausen L, Thébault E et al (2010) International geomagnetic reference field: the eleventh generation. Geophys I Int 183(3):1216-1230. doi: 10.1111/j.1365-246X.2010.04804.X.

Fournier A, Hulot G, Jault D, Kuang W, Tangborn A, Gillet N, Canet E, Aubert J, Lhuillier F (2010) An introduction to data assimilation and predictability in geomagnetism. Space Sci Rev 155:247-291. doi: 10.1007/s11214-010-9669-4.

Fournier A, Aubert J, Thébault E (2015) A candidate secular variation model for IGRF-12 based on Swarm data and inverse geodynamo modelling. Earth Planets Space 67:81 doi: 10.1186/s40623-015-0245-8.

Friis-Christensen E, Lühr H, Hulot G (2006) Swarm: a constellation to study the Earth's magnetic field. Earth Planets Space 58:351-358. doi: 10.1186/ BF03351933.

Gravrand O, Khokhlov A, Le Mouël JL, Léger JM (2001) On the calibration of a vectorial 4 He pumped magnetometer. Earth Planets Space 53:949-958. doi: 10.1186/BF03351692.

Holme R, Bloxham J (1996) The treatment of attitude errors in satellite geomagnetic data. Phys Earth Planet Int 98:221-233. doi: 10.1016/S00319201(96)03189-5.

Hulot G, Sabaka TJ, Olsen N, Fournier A (2015a) The present and future geomagnetic field. In: Kono M (ed), Geomagnetism. Treatise on Geophysics, vol 5, 2nd edn. Elsevier, Amsterdam. doi: 10.1016/B978-0-444-53802-4.00096-8.

Hulot G, Vigneron P, Léger J-M, Fratter I, Olsen N, Jager T, Bertrand F, Brocco L, Sirol O, Lalanne X, Boness A, Cattin V (2015b) Swarm's absolute magnetometer experimental vector mode, an innovative capability for space magnetometry. Geophys Res Lett 42:1352-1359. doi: 10.1002/2014GL062700.

Léger JM, Bertrand F, Jager T, Prado ML, Fratter I, Lalaurie JC (2009) Swarm absolute scalar and vector magnetometer based on helium 4 optical pumping. Procedia Chemistry 1:634-637. doi: 10.1016/j.proche.2009.07.158.

Léger JM, Jager T, Bertrand F, Hulot G, Brocco L, Vigneron P, Lalanne X, Chulliat A, Fratter I (2015) In-flight performances of the absolute scalar magnetometer vector mode on board the Swarm satellites. Earth Planets Space 67:57. doi: 10.1186/s40623-015-0231-1.

Lowes FJ (1966) Mean-square values on sphere of spherical harmonic vector fields. J Geophys Res 71:2179

Macmillan S, Finlay C (2011) The international geomagnetic reference field. In: Mandea M, Korte M (eds) Geomagnetic observations and models, vol 5, IAGA Special Sopron Book Series. Springer, Heidelberg, pp 265-76

Mauersberger P (1956) Das Mittel der Energiedichte des geomagnetischen Hauptfeldes an der Erdoberfläche und seine säkulare Änderung. Gerl Beitr Geophys 65:207-15

Olsen N, Lühr H, Finlay CC, Sabaka TJ, Michaelis I, Rauberg J, Tøffner-Clausen L (2014) The CHAOS-4 geomagnetic field model. Geophys J Int 197:815-27

Olsen N, Hulot G, Lesur V, Finlay CC, Beggan C, Chulliat A, Sabaka TJ, Floberghagen R, Friis-Christensen E, Haagmans R, Kotsiaros S, Lühr H, Tøffner-Clausen L, Vigneron P (2015) The Swarm initial field model for the 2014 geomagnetic field. Geophys Res Lett 42:1092-1098. doi:10.1002/ 8512014 GL062659

Richmond AD (1995) lonospheric electrodynamics using magnetic Apex coordinates. J Geomagn Geoelectr 47:191-212

Thébault E, Finlay CC, Beggan C, Alken P, Aubert J, Barrois O, Bertrand F, Bondar T, Boness A, Brocco L, Canet E, Chambodut A, Chulliat A, Coïsson P, Civet F, Du A, Fournier A, Fratter I, Gillet N, Hamilton B, Hamoudi M, Hulot G, Jager T, Korte M, Kuang W, Lalanne X, Langlais B, Léger J-M, Lesur V, Lowes FJ, Macmillan S, Mandea M, Manoj C, Maus S, Olsen N, Petrov V, Rother M, Sabaka TJ, Saturnino D, Schachtschneider R, Sirol O, Tangborn A, Taylor V, Thomson A, Tøffner-Clausen L, Vigneron P, Wardinski I, Zvereva T (2015a) International Geomagnetic Reference Field: the twelfth generation. Earth Planets Space 67:79. doi: 10.1186/s40623-015-0228-9.

Thébault E., Finlay CC, Alken P, Beggan C, Canet E, Chulliat A, Manoj C, Langlais B, Lesur V, Lowes FJ, Petrov M, Rother M, Schachtschneider R (2015b) Evaluation of candidate geomagnetic field models for IGRF-12. Earth Planets Space, in press 